NYU-TH/00/04/01

April 25, 2000

\title{
4D Gravity on a Brane in 5D Minkowski Space
}

\author{
Gia Dvali, Gregory Gabadadze, Massimo Porrati \\ Department of Physics, New York University, New York, NY 10003
}

\begin{abstract}
We suggest a mechanism by which four-dimensional Newtonian gravity emerges on a 3-brane in 5D Minkowski space with an infinite size extra dimension. The worldvolume theory gives rise to the correct $4 \mathrm{D}$ potential at short distances whereas at large distances the potential is that of a 5D theory. We discuss some phenomenological issues in this framework.
\end{abstract}




\section{Introduction}

The observed weakness of gravity may be due to the fact that we live on a brane embedded in space with large extra dimensions [1]. The correct 4D gravity can be reproduced at large distances due to the finite volume of extra space. This is usually achieved by compactifying extra space. Alternatively, this can be obtained by keeping extra space uncompactified but warped as in the scenario of [2], where the size $L$ of extra space is still finite $\left(L=2 \int_{0}^{\infty} \sqrt{g} d y<\infty\right)$.

In this work we shall discuss the fate of $4 \mathrm{D}$ gravity in theories with infinite size flat extra dimensions. These models may shed new light on supersymmetry breaking and the cosmological constant problem [3, 4], since they make compatible

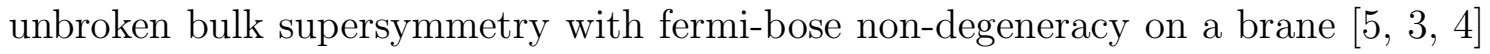
(for related discussions, see [6]). A first example with an infinite size extra dimension and the correct 4D Newtonian potential was proposed in [7] as a generalization of the scenario of [2]. However, the model of [7] has ghosts 1 .

In the present paper we suggest a general mechanism by which 4D Newtonian gravity may be generated on any static 3-brane embedded in 5D Minkowski space. We show that the $4 \mathrm{D}$ scalar curvature term in the worldvolume brane action

$$
M_{P}^{2} \int d^{4} x \sqrt{|g|} R,
$$

can be responsible for the correct 4D Newtonian interaction on a brane, despite of the fact that gravity propagates in 5D Minkowski space. Such a term is compatible with all the symmetries left unbroken on a brane and can be included in the theory. Moreover, if it is absent in a classical theory, it may be generated on a brane by quantum corrections. It turns out that inclusion of such a term automatically generates the $1 / r$ gravitational potential at short distances for the sources localized on a brane. As a result, an observer on a brane will see correct Newtonian gravity despite of the fact that gravity propagates in extra space which is flat and has the infinite extent. We should note here that the modification of gravity on a brane due to this effect in weakly coupled string theory would take place at very short, phenomenologically unacceptable distances. Nevertheless, it is interesting to study this mechanism in a low-energy field theory framework.

The paper is organized as follows. In section 2 we present a 5D model with an infinite size extra dimension and discuss mechanisms by which 4D Newtonian gravity on a brane can be obtained from higher dimensional theory. In section 3

${ }^{1}$ The 4D Newtonian force in [7] is mediated by a resonance graviton [7, 8, 3] which has extra polarization degrees of freedom [3]. The brane construction of [7] violates the null-energy condition [1], and, as a result, it has a state with negative norm, a ghost [9, 10, 11, 12]. A brane bending term 13 which emerges in the traceless-transverse gauge, is a manifestation of a ghost 9]. In the harmonic gauge the ghost can be identified with the radion field which has a negative kinetic term [12]. This ghost can cancel the unwanted polarizations of a resonance graviton [13, 14, 12]. However, the presence of a propagating ghost signals the inconsistency [9, 12] of the model of Ref. [7]. 
we study a prototype model which involves only a scalar field and calculate the potential which is produced by mediation of this scalar. At short distances the potential has $4 \mathrm{D}$ nature, while it has $5 \mathrm{D}$ behavior at large distances. In section 4 we study the $4 \mathrm{D}$ graviton propagator in the infinite extra dimensional model. As in the case with a scalar, gravitons mediate the 4D Newton potential at small distances with a crossover to the 5D potential at large distances. Discussions of some phenomenological issues are given in section 5 .

\section{A Model of 4D Gravity in 5D Minkowski Space}

We start with a $D=(4+1)$ dimensional theory. Let us suppose there is a 3brane embedded in 5-dimensional space-time. We assume that this is a zero-tension brane in 5-dimensions. The four coordinates of our world are $x_{\mu}, \mu=0,1,2,3$; the extra coordinate will be denoted by $y$. Capital letters and subscripts will be used for $5 \mathrm{D}$ quantities $(A, B, C=0,1,2,3,5)$; the metric convention is mostly negative.

Let us consider the action:

$$
S=M^{3} \int d^{5} X \sqrt{G} \mathcal{R}_{(5)}+M_{P}^{2} \int d^{4} x \sqrt{|g|} R
$$

where $M$ stands for the $5 \mathrm{D}$ Planck mass, and $M_{P}$ is the $4 \mathrm{D}$ Planck mass; as they stand in (11) $M$ and $M_{P}$ are independent (in general they can be dependent). $G_{A B}(X) \equiv G_{A B}(x, y)$ denotes a $5 \mathrm{D}$ metric for which the 5D Ricci scalar is $\mathcal{R}_{(5)}$. The brane is located at $y=0$. The induced metric on the brane is denoted by

$$
g_{\mu \nu}(x) \equiv G_{\mu \nu}(x, y=0)
$$

The 4D Ricci scalar for $g_{\mu \nu}(x)$ is $R=R(x)$. Possible additional terms of the corresponding SUGRA and/or matter fields confined to a brane are omitted in Eq. (1) for simplicity.

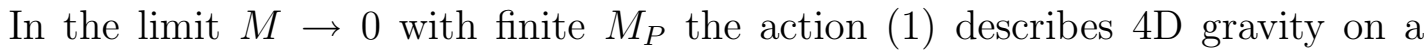
brane. On the other hand, in the limit $M_{P} \rightarrow 0$ with finite $M$ it describes $5 \mathrm{D}$ bulk gravity. In what follows we study $4 \mathrm{D}$ gravitational interactions on a brane when both $M$ and $M_{P}$ are finite.

Before we turn to these discussions let us try to understand a possible origin of the action (11).

The first possibility is related to the fact that in certain cases (to be specified below) the localized matter on a brane can induce via loop corrections a $4 \mathrm{D}$ kinetic term for gravitons. To demonstrate this, suppose there are matter fields confined to a brane. Thus, the matter energy-momentum tensor can be written as follows:

$$
T_{A B}=\left(\begin{array}{cc}
T_{\mu \nu}(x) \delta(y) & 0 \\
0 & 0
\end{array}\right) .
$$




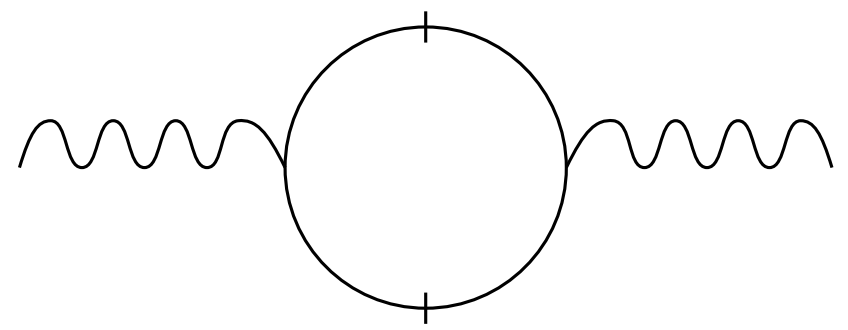

Figure 1: The one-loop diagram generating the Ricci scalar for $4 \mathrm{D}$ graviton. Wave lines denote gravitons, solid lines denote massive scalars/fermions. Vertical short lines on scalar/fermion propagators indicate that they are massive.

As a result, the interaction Lagrangian of localized matter with 5D metric fluctuations $h_{A B}(x, y) \equiv G_{A B}(x, y)-\eta_{A B}$, reduces to the following expression:

$$
\mathcal{L}_{\mathrm{int}}=\int d y h^{\mu \nu}(x, y) T_{\mu \nu}(x) \delta(y)=h^{\mu \nu}(x, 0) T_{\mu \nu}(x)
$$

where the $4 \mathrm{D}$ induced metric $g_{\mu \nu}(x)=\eta_{\mu \nu}+h_{\mu \nu}$ is defined as in (2). Due to this interaction, a 4D kinetic term can be generated for $g_{\mu \nu}(x)$ in the full quantum theory. For instance, the diagram of Fig. 1 with massive scalars [15], or fermions [16, 17] running in the loop would induce the following $4 \mathrm{D}$ term in the low-energy action:

$$
\int d^{4} x d y \delta(y) \sqrt{|g|} R
$$

The corresponding induced gravitational constant will be determined by a correlation function of the world-volume matter theoryf. The magnitude of this constant depends on a worldvolume theory at hand and is vanishing in conformaly invariant models, or nonzero if conformal invariance is broken (for detailed discussions see [16, 17, 18]). We will not attempt to discuss these model dependent features here, we rather assume that the worldvolume theory is such that the second term in (四) is generated with a proper sing and magnitude. We also neglect the induced 4D cosmological constant (which renormalizes the brane tension), $\Lambda=\left\langle 0\left|T_{\mu}^{\mu}\right| 0\right\rangle$, as well as higher derivative terms $\mathcal{O}\left(R^{2}\right)$ which can be generated in this case as well. The induced 4D cosmological constant, in general, should be canceled by the brane tension and/or by the vacuum energy due to light matter fields on a brane. Without SUSY this is a usual fine tuning. However, if the bulk is supersymmetric (even though SUSY is broken on a brane) it might require the exact cancellation of the total 4D cosmological constant in a model which at large distances (low energies) becomes

\footnotetext{
${ }^{2}$ For instance, in the 4D framework of Refs. 16, 17]:

$$
M_{P}^{2}=\frac{i}{96} \int d^{4} x x^{2}\left\{\langle T S(x) S(0)\rangle-\langle S\rangle^{2}\right\}
$$

where $S(x) \equiv T_{\mu}^{\mu}$ is the trace of the energy-momentum tensor of $4 \mathrm{D}$ states running in the loop.
} 
effectively 5-dimensional. This certainly needs further separate investigation. Here we assume that the total 4D cosmological constant is zero.

The second way to think of the origin of the $4 \mathrm{D}$ term in (11) is to imagine that $5 \mathrm{D}$ gravity is coupled to a certain 5D scalar field $\phi$. Suppose that in addition to usual terms there are terms in which $\phi$ couples to the five-dimensional Ricci scalar. Furthermore, suppose that the scalar field potential is such that there is a kink type solution to its equations of motion: $\phi_{\text {classical }}=v \tanh (v y)$. This background, due to the presence of higher derivative interactions might produce the 4D Ricci scalar which would be peaked around the point $y=0$. In the limit of a zero width for the kink this term can be approximated by (5). A prototype example with scalars will be presented in the next section. Then we turn to the consideration of gravitons.

\section{A Simple Example with Scalars}

In this section we study an instructive example which involves a single 5D scalar field. We write the action of the model as follows:

$$
S=M^{3} \int d^{4} x d y \partial_{A} \Phi(x, y) \partial^{A} \Phi(x, y)+M_{P}^{2} \int d^{4} x d y \delta(y) \partial_{\mu} \Phi(x, 0) \partial^{\mu} \Phi(x, 0) .
$$

For the purpose of comparison with gravity we choose to work with the unconventionally normalized dimensionless scalar field $\Phi$. Let us notice that this kind of action could arise if the 5D field $\Phi$ is placed in the background $\chi=v \tanh (v y)$, and the interactions between these fields are taken as: $\left(\partial_{A} \Phi\right)^{2}+\left(\partial_{A} \chi\right)^{2}\left(\partial_{B} \Phi\right)^{2}-\left(\partial_{A} \Phi \partial^{A} \chi\right)^{2}+\ldots$ This leads to the equation of motion for $\Phi$ : $2 \partial_{A}^{2} \Phi+\left(\chi^{\prime}\right)^{2} \partial_{\mu}^{2} \Phi=0$. Here the prime denotes derivative with respect to $y . \quad\left(\chi^{\prime}\right)$ is a peaked function at $y=0$ point. Therefore, this equation can be modeled by the action (6).

Our goal is to determine the distance dependence of interactions which are mediated by this scalar in a $4 \mathrm{D}$ worldvolume theory. For this we should find the corresponding retarded Green function and calculate the potential. The classical equation for the Green function looks as follows:

$$
\left(M^{3} \partial_{A} \partial^{A}+M_{P}^{2} \delta(y) \partial_{\mu} \partial^{\mu}\right) G_{R}(x, y ; 0,0)=\delta^{(4)}(x) \delta(y),
$$

where $G_{R}(x, y ; 0,0)=0$ for $x_{0}<0$.

The potential mediated by the scalar $\Phi$ on the $4 \mathrm{D}$ worldvolume of the brane is determined as:

$$
V(r)=\int G_{R}(t, \vec{x}, y=0 ; 0,0,0) d t
$$

where $r \equiv \sqrt{x_{1}^{2}+x_{2}^{2}+x_{3}^{2}}$. To find a solution of (7) let us turn to Fourier-transformed quantities with respect to the worldvolume four-coordinates $x_{\mu}$ :

$$
G_{R}(x, y ; 0,0) \equiv \int \frac{d^{4} p}{(2 \pi)^{4}} e^{i p x} \tilde{G}_{R}(p, y) .
$$


Turning to Euclidean space the equation (7) takes the form:

$$
\left(M^{3}\left(p^{2}-\partial_{y}^{2}\right)+M_{P}^{2} p^{2} \delta(y)\right) \tilde{G}_{R}(p, y)=\delta(y)
$$

Here $p^{2}$ denotes the square of an Euclidean four-momentum. The solution with appropriate boundary conditions takes the form:

$$
\tilde{G}_{R}(p, y)=\frac{1}{M_{P}^{2} p^{2}+2 M^{3} p} \exp (-p|y|)
$$

where $p \equiv \sqrt{p^{2}}=\sqrt{p_{4}^{2}+p_{1}^{2}+p_{2}^{2}+p_{3}^{2}}$. Using this expression and Eq. (\$) one finds the following (properly normalized) formula for the potential mediated by a scalar in $4 \mathrm{D}$ brane worldvolume:

$$
V(r)=-\frac{1}{8 \pi^{2} M_{P}^{2}} \frac{1}{r}\left\{\sin \left(\frac{r}{r_{0}}\right) \mathrm{Ci}\left(\frac{r}{r_{0}}\right)+\frac{1}{2} \cos \left(\frac{r}{r_{0}}\right)\left[\pi-2 \operatorname{Si}\left(\frac{r}{r_{0}}\right)\right]\right\},
$$

where $\operatorname{Ci}(z) \equiv \gamma+\ln (z)+\int_{0}^{z}(\cos (t)-1) d t / t, \operatorname{Si}(z) \equiv \int_{0}^{z} \sin (t) d t / t, \gamma \simeq 0.577$ is the Euler-Masceroni constant, and the distance scale $r_{0}$ is defined as follows:

$$
r_{0} \equiv \frac{M_{P}^{2}}{2 M^{3}}
$$

It is useful to study the short distance and long distance behavior of this expression.

At short distances when $r<<r_{0}$ we find:

$$
V(r) \simeq-\frac{1}{8 \pi^{2} M_{P}^{2}} \frac{1}{r}\left\{\frac{\pi}{2}+\left[-1+\gamma+\ln \left(\frac{r}{r_{0}}\right)\right]\left(\frac{r}{r_{0}}\right)+\mathcal{O}\left(r^{2}\right)\right\}
$$

As we expected, at short distances the potential has the correct $4 \mathrm{D}$ Newtonian $1 / r$ scaling. This is subsequently modified by the logarithmic repulsion term in (14).

Let us turn now to the large distance behavior. Using (12) we obtain for $r>>r_{0}$ :

$$
V(r) \simeq-\frac{1}{8 \pi^{2} M_{P}^{2}} \frac{1}{r}\left\{\frac{r_{0}}{r}+\mathcal{O}\left(\frac{1}{r^{2}}\right)\right\}
$$

Thus, the long distance potential scales as $1 / r^{2}$ in accordance with laws of $5 \mathrm{D}$ theory. These properties are similar to those obtained in the model of Ref. [7].

In the next section we consider the system defined in (11). We will show that the short and long distance behavior of the Newtonian potential for (1) is determined by the scalar example studied in this section.

\section{Gravitational potential}

Based on the scalar field example discussed in the previous section we expect that the system (11) will produce the $1 / r$ gravitational potential at short distances 
and $1 / r^{2}$ potential at large scales. Therefore, if $r_{0}$ in (13) is big enough there will be no contradictions with Newtonian predictions. However, there is a subtlety when it comes to relativistic effects. This is related to the structure of the graviton propagator. The tensor structure of a $4 \mathrm{D}$ massless graviton propagator looks as follows (we omit momentum-dependent parts):

$$
\frac{1}{2} \eta^{\mu \alpha} \eta^{\nu \beta}+\frac{1}{2} \eta^{\mu \beta} \eta^{\nu \alpha}-\frac{1}{2} \eta^{\mu \nu} \eta^{\alpha \beta}
$$

while for a 4D massive case, or equivalently for a 5D massless case, it takes the following form:

$$
\frac{1}{2} \eta^{\mu \alpha} \eta^{\nu \beta}+\frac{1}{2} \eta^{\mu \beta} \eta^{\nu \alpha}-\frac{1}{3} \eta^{\mu \nu} \eta^{\alpha \beta}
$$

The difference in the last coefficient ( $1 / 2$ versus $1 / 3$ ) is vital for description of observations. It was shown in Refs. [19, 20] that no matter how small the graviton mass is in 4D, due to the difference in the tensor structures in (16) and (17), predictions for bending of light are substantially different in the two cases. Moreover, the structure (17) gives contradictions with observations. Any theory with massive gravitons, no matter how light they are, will have to face this problem since there is a discontinuity in the limit when the graviton mass is taken to zero. This can easily be understood in terms of degrees of freedom: a massive graviton has 5 degrees of freedom 3 of which couple to a conserved energy-momentum tensor. Thus, having the propagator as in (17) is equivalent of having a tensor-scalar gravity from 4D point of view. This extra scalar polarization degree of freedom yields additional attractive force. We will continue this discussion in the next section after we find out what is the correct tensor structure of the graviton propagator in the model (四).

To find the propagator let us introduce the metric fluctuations:

$$
G_{A B}=\eta_{A B}+h_{A B}
$$

We choose the harmonic gauge in the bulk:

$$
\partial^{A} h_{A B}=\frac{1}{2} \partial_{B} h_{C}^{C}
$$

It can be checked that the choice

$$
h_{\mu 5}=0
$$

is consistent with the equations of motions for (11). Thus, the surviving components of $h_{A B}$ are $h_{\mu \nu}$ and $h_{55}$. In this gauge the (55) component of Einstein's equations yields:

$$
\partial_{\mu} \partial^{\mu} h_{\nu}^{\nu}=\partial_{\mu} \partial^{\mu} h_{5}^{5}
$$


which combined with the gauge fixing conditions (19, 20) implies:

$$
\partial_{A} \partial^{A} h_{\nu}^{\nu}=\partial_{B} \partial^{B} h_{5}^{5}
$$

Subscripts and superscripts in all these equations are raised and lowered by a flat space metric tensor. Finally, we come to the $(\mu \nu)$ components of the Einstein equation for (1). After some rearrangements it takes the form:

$$
\begin{aligned}
\left(M^{3} \partial_{A} \partial^{A}+M_{P}^{2} \delta(y) \partial_{\mu} \partial^{\mu}\right) h_{\mu \nu}(x, y)= & \left\{T_{\mu \nu}-\frac{1}{3} \eta_{\mu \nu} T_{\alpha}^{\alpha}\right\} \delta(y) \\
& +M_{P}^{2} \delta(y) \partial_{\mu} \partial_{\nu} h_{5}^{5}
\end{aligned}
$$

This has a structure of a massive 4D graviton or, equivalently that of a massless 5D graviton, indicating that the tensor structure of the propagator looks as in (17). In this respect, it is instructive to rewrite the expression (23) in the following form:

$$
\begin{array}{r}
\left(M^{3} \partial_{A} \partial^{A}+M_{P}^{2} \delta(y) \partial_{\mu} \partial^{\mu}\right) h_{\mu \nu}(x, y)=\left\{T_{\mu \nu}-\frac{1}{2} \eta_{\mu \nu} T_{\alpha}^{\alpha}\right\} \delta(y) \\
-\frac{1}{2} M^{3} \eta_{\mu \nu} \partial_{A} \partial^{A} h_{\alpha}^{\alpha}+M_{P}^{2} \delta(y) \partial_{\mu} \partial_{\nu} h_{5}^{5} .
\end{array}
$$

Here the tensor structure on the r.h.s. is that of a 4D massless graviton (16). However, there is an additional contribution due to the trace part $h_{\mu}^{\mu}$ which is nonzero. Therefore, one is left with the theory of gravity which from the $4 \mathrm{D}$ point of view is mediated by a graviton plus a scalar

Let us now present the exact form for the graviton propagator of (1). Turning to the Fourier images in the Euclidean space as in the previous section we find:

$$
\tilde{h}_{\mu \nu}(p, y=0) \tilde{T}^{\mu \nu}(p)=\frac{\tilde{T}^{\mu \nu} \tilde{T}_{\mu \nu}-\frac{1}{3} \tilde{T}_{\mu}^{\mu} \tilde{T}_{\nu}^{\nu}}{M_{P}^{2} p^{2}+2 M^{3} p} .
$$

Here the tilde sign denotes the Fourier-transformed quantities. Thus, the tensor structure of the graviton propagator in $4 \mathrm{D}$ worldvolume theory looks as follows:

$$
D^{\mu \nu \alpha \beta}=\frac{1}{2} \eta^{\mu \alpha} \eta^{\nu \beta}+\frac{1}{2} \eta^{\mu \beta} \eta^{\nu \alpha}-\frac{1}{3} \eta^{\mu \nu} \eta^{\alpha \beta}+\mathcal{O}(p)
$$

At short distances the potential scales as $1 / r$ with the logarithmic corrections defined in (14). On the other hand, at large distances the $1 / r^{2}$ behavior is recovered in (15). The tensor structure of the propagator is that of $4 \mathrm{D}$ tensor-scalar gravity.

\footnotetext{
${ }^{3}$ The same result could be obtained by using the traceless-transverse gauge and taking into account the "brane bending" effects 21, 22]. We would like to emphasize here that the change of the coefficient $1 / 3$ to the coefficient $1 / 2$ in [21, 22] is due to the possibility to perform gauge transformations of $h_{\mu \nu}$ which generate terms proportional to $\eta_{\mu \nu}$. This term appears due to the nontrivial warp factor. In the present case the warp-factor is absent, thus the terms proportional to $\eta_{\mu \nu}$ are absent in gauge transformations and the change of the coefficient $1 / 3$ into $1 / 2$ does not take place.
} 


\section{Discussions and Outlook}

So far we worried about the theoretical consistency of the model with an infinite size extra dimension (1). In this section we address the issue whether this model could be phenomenologically viable. There are two outstanding questions in this regard. The first one deals with the extra degree of freedom which shows up in the propagator (26): one should wonder if it is possible to cancel it.

The second question concerns the modification of the Newton law by logarithmic corrections (14): what is the distance at which these modifications will be harmless?

We will discuss these two issues in turn. Let us start with the extra degree of freedom. As we said above, there is additional attraction in the theory due to the extra scalar mode. We should look for some new states which could compensate for this extra attraction. This can certainly be done by a ghost field which gives rise to repulsive force. However, it is hard to make sense of a theory with a manifest ghost. The ghost could be produced as a final state in various processes and this would ruin the consistency of the model at hand. Thus, we should exclude the possibility of using a ghost and try to utilize some other means.

A possibility to compensate for additional attraction would be to use an exchange of a vector particle. One could think of a scenario where all matter fields are given additional $U(1)$ charges so that the corresponding gauge fields lead to repulsive interactions. Then one should tune the parameters of the model so that this repulsion cancels (at least partially) the additional attraction in (26). Whether this scenario suffices a single extra $U(1)$ or needs a few of them (so that the possible equivalence principle violation is not observable) should be determined by separate phenomenological studies. It is also possible that there exists a more elegant solution to this problem.

Let us now turn to the second issue, what is the crossover scale where gravity in (11) changes its behavior? As we discussed in section 3 this scale is determined by the ratio $r_{0}=M_{P}^{2} / 2 M^{3}$. Taking the value of the four-dimensional Planck scale $M_{P} \simeq 10^{19} \mathrm{GeV}$ and assuming that the five dimensional Planck scale is in the $\mathrm{TeV}$ region, we find that $r_{0} \sim 10^{15} \mathrm{~cm}$. This is precisely the size of the solar system. However, the crossover scale should be much bigger than the solar system size. For instance, requiring that the logarithmic corrections in (14) give the effects which are small compared to the General Relativity corrections to the precession of the Mercury perihelion, one obtains that the crossover scale should be about $10^{8}$ times bigger then the solar system size. This means that $M$ should be about $10^{2}-10^{3}$ times smaller then the $\mathrm{TeV}$ scalef. It is not obvious at present whether such a low scale can be obtained from higher dimensional theories.

In conclusion, we suggested a class of models in which $4 \mathrm{D}$ Newtonian gravity can emerge on a brane in 5D flat space. A crucial feature of these models is that

\footnotetext{
${ }^{4}$ As we mentioned before, in non-supersymmetric D-brane constructions within weakly coupled string theory induced $4 \mathrm{D}$ terms will modify gravity at distances less than the string scale.
} 
the extra dimension is neither compact nor warped and its size is truly infinite. The modification of the Newton law takes place at large distances in this framework. In the minimal setup there are phenomenological subtleties. We outlined some possible ways to circumvent them by adding extra fields. More detailed studies are needed in order determine whether models with infinite size extra dimensions can be given a complete, phenomenologically viable form.

We would like to emphasize that the similar mechanism can be used for "localization" of scalar (discussed in section 3) and vector fields on a brane in flat higher dimensional space. In these cases, the phenomenological subtleties which were present for gravitons are gone. As a result, one is left with theoretically, as well as phenomenologically consistent models of localization for spin-0 and spin-1 fields.

\section{Acknowledgments}

The work of G.D. is supported in part by a David and Lucile Packard Foundation

Fellowship for Science and Engineering. G.G. is supported by NSF grant PHY-9423002. M.P. is supported in part by NSF grant PHY-9722083.

\section{References}

[1] N. Arkani-Hamed, S. Dimopoulos, G. Dvali, Phys. Lett. B429, 263 (1998); Phys. Rev. D59, 0860 (1999); I. Antoniadis, N. Arkani-Hamed, S. Dimopoulos, G. Dvali, Phys. Lett. B436, 257 (1998).

[2] L. Randall, R. Sundrum, Phys. Rev. Lett. 83, 3370 (1999) ; Phys. Rev. Lett. 83, 4690 (1999) .

[3] G. Dvali, G. Gabadadze, M. Porrati, hep-th/0002190 .

[4] E. Witten, hep-ph/0002297.

[5] G. Dvali, M. Shifman, Nucl. Phys. B504, 127 (1997).

[6] G. Dvali, hep-th/0004057 .

[7] R. Gregory, V.A. Rubakov, S.M. Sibiryakov, hep-th/0002072 .

[8] C. Csáki, J. Erlich, T.J. Hollowood, hep-th/0002161] .

[9] G. Dvali, G. Gabadadze, M. Porrati, hep-th/0003054 .

[10] I. Kogan, G. G. Ross, hep-th/0003074 .

[11] G. Kang, Y.S. Myung, hep-th/0003162 .

[12] L. Pilo, R. Rattazzi, A. Zaffaroni, hep-th/0004028] . 
[13] C. Csáki, J. Erlich, T.J. Hollowood, hep-th/0003020 .

[14] R. Gregory, V.A. Rubakov, S.M. Sibiryakov, hep-th/0003045 .

[15] D.M. Capper, Nuovo Cim. A25 29 (1975) .

[16] S. L. Adler, Phys. Rev. Lett. 441567 (1980) ; Phys. Lett. B95 241 (1980) ; Rev. Mod. Phys. 54729 (1982); Erratum-ibid. 55837 (1983).

[17] A. Zee, Phys. Rev. Lett. 48295 (1982).

[18] N.N. Khuri, Phys. Rev. Lett. 49513 (1982) ; Phys. Rev. D26 2664 (1982).

[19] H. van Dam, M. Veltman, Nucl. Phys. B22, 397 (1970) .

[20] V.I. Zakharov, JETP Lett. 12, 312 (1970) .

[21] J. Garriga, T. Tanaka, hep-th/9911055

[22] S.B. Giddings, E. Katz, L. Randall, hep-th/0002091 . 